\title{
Optimally Accurate Second-Order Time-Domain Finite-Difference Scheme for Acoustic, Electromagnetic, and Elastic Wave Modeling
}

\author{
C. Bommaraju, R. Marklein, and P. K. Chinta
}

Fachgebiet Theoretische Elektrotechnik, Fachbereich Elektrotechnik/Informatik, Universität Kassel (UNIK), 34109 Kassel, Germany

\begin{abstract}
Numerical methods are extremely useful in solving real-life problems with complex materials and geometries. However, numerical methods in the time domain suffer from artificial numerical dispersion. Standard numerical techniques which are second-order in space and time, like the conventional Finite Difference 3-point (FD3) method, FiniteDifference Time-Domain (FDTD) method, and Finite Integration Technique (FIT) provide estimates of the error of discretized numerical operators rather than the error of the numerical solutions computed using these operators. Here optimally accurate time-domain FD operators which are secondorder in time as well as in space are derived. Optimal accuracy means the greatest attainable accuracy for a particular type of scheme, e.g., second-order FD, for some particular grid spacing. The modified operators lead to an implicit scheme. Using the first order Born approximation, this implicit scheme is transformed into a two step explicit scheme, namely predictor-corrector scheme. The stability condition (maximum time step for a given spatial grid interval) for the various modified schemes is roughly equal to that for the corresponding conventional scheme. The modified FD scheme (FDM) attains reduction of numerical dispersion almost by a factor of 40 in 1-D case, compared to the FD3, FDTD, and FIT. The CPU time for the FDM scheme is twice of that required by the FD3 method. The simulated synthetic data for a 2-D P-SV (elastodynamics) problem computed using the modified scheme are 30 times more accurate than synthetics computed using a conventional scheme, at a cost of only 3.5 times as much CPU time. The FDM is of particular interest in the modeling of large scale (spatial dimension is more or equal to one thousand wave lengths or observation time interval is very high compared to reference time step) wave propagation and scattering problems, for instance, in ultrasonic antenna and synthetic scattering data modeling for Non-Destructive Testing (NDT) applications, where other standard numerical methods fail due to numerical dispersion effects. The possibility of extending this method to staggered grid approach is also discussed. The numerical FD3, FDTD, FIT, and FDM results are compared against analytical solutions.
\end{abstract}

Correspondence to: R. Marklein

(marklein@uni-kassel.de)

\section{Introduction}

Numerical methods in the time-domain suffer from artificial numerical dispersion. Standard numerical techniques, like Finite-Difference Time-Domain (FDTD) method (Taflove and Hagness, 2000) and Finite Integration Technique (FIT) (Marklein, 1997) provide estimates of the error of discretized numerical operators rather than the error of the numerical solutions computed using these operators (Geller and Takeuchi, 1995, 1998; Bommaraju and Marklein, 2004). Optimal accuracy means the greatest attainable accuracy for a particular type of scheme, e.g., second-order FD, for some particular grid spacing.

\section{Modified operators for the 1-D case}

Consider the homogeneous, source-free 1-D electromagnetic (EM) wave equation in the time domain

$\left(\varepsilon_{0} \frac{\partial^{2}}{\partial t^{2}}-\frac{1}{\mu_{0}} \frac{\partial^{2}}{\partial z^{2}}\right) E_{x}(z, t)=0$,

where $E_{x}$ represents the electric field strength, $\varepsilon_{0}$ and $\mu_{0}$ are the free-space permittivity and permeability, respectively. Let $A_{n, n_{t}}$ be the discretized second-order Finite Difference (FD) operator for $\varepsilon_{0} \frac{\partial^{2}}{\partial t^{2}}$, and $K_{n, n_{t}}$ be the FD operator for $\frac{1}{\mu_{0}} \frac{\partial^{2}}{\partial z^{2}}$ and $c_{n, n_{t}}$ represents the unknown discrete $E_{x}\left(N \Delta z, N_{t} \Delta t\right)$ component. The above equation can be represented as

$$
\left(A_{n, n_{t}}-K_{n, n_{t}}\right) c_{n, n_{t}}=0 .
$$

Following the FD3 method, $A_{n, n_{t}}$ and $K_{n, n_{t}}$ have the numerical dispersion of normal three point second derivative operators. The superscript " 0 " indicates the conventional FD (FD3) operators. Thus, omitting higher order terms, the operator error for the FD3 method read

$$
\begin{aligned}
& \left(\delta A_{n, n_{t}}^{0}-\delta K_{n, n_{t}}^{0}\right) c_{n, n_{t}}= \\
& \left.\varepsilon_{0} \frac{(\Delta t)^{2}}{12}\left[\frac{\partial^{4} E_{x}(z, t)}{\partial t^{4}}\right]\right|_{n, n_{t}}-\left.\frac{1}{\mu_{0}} \frac{(\Delta z)^{2}}{12}\left[\frac{\partial^{4} E_{x}(z, t)}{\partial z^{4}}\right]\right|_{n, n_{t}} .
\end{aligned}
$$


The relation between the exact and conventional operators can be expressed as

$$
\begin{aligned}
& A_{n, n_{t}}^{\text {exact }}=A_{n, n_{t}}^{0}-\delta A_{n, n_{t}}^{0} \\
& K_{n, n_{t}}^{\text {exact }}=K_{n, n_{t}}^{0}-\delta K_{n, n_{t}}^{0} .
\end{aligned}
$$

The transformation into the frequency domain yields

$$
\begin{gathered}
\left(\delta A_{n, n_{\omega}}-\delta K_{n, n_{\omega}}\right) c_{n, n_{\omega}}=\left.\frac{\omega^{2}(\Delta t)^{2}}{12}\left[\varepsilon_{0} \omega^{2} E_{x}(z, \omega)\right]\right|_{n, n_{\omega}} \\
+\left.\frac{(\Delta z)^{2}}{12} \frac{\partial^{2}}{\partial z^{2}}\left[\frac{1}{\mu_{0}} \frac{\partial^{2}}{\partial z^{2}} E_{x}(z, \omega)\right]\right|_{n, n_{\omega}},
\end{gathered}
$$

where $\delta A_{n, n_{\omega}}^{0}, \delta K_{n, n_{\omega}}^{0}, c_{n, n_{\omega}}$ are the frequency domain representations of $\delta A_{n, n_{t}}^{0}, \delta K_{n, n_{t}}^{0}$, and $c_{n, n_{t}}$, respectively. The quantity on the right-hand side of the above equation is the basic error. When $E_{x}$ is an eigenfunction and $\omega$ approaches the corresponding eigenfrequency the basic error will not in general equal zero. Instead we have

$$
\begin{aligned}
& \left(\delta A_{n, n_{\omega}}-\delta K_{n, n_{\omega}}\right) c_{n, n_{\omega}}= \\
& \left.\frac{\omega^{2}(\Delta t)^{2}}{12}\left[\varepsilon_{0} \omega^{2} E_{x}(z, \omega)+\frac{1}{\mu_{0}} \frac{\partial^{2}}{\partial z^{2}} E_{x}(z, \omega)\right]\right|_{n, n_{\omega}} \\
& +\left.\frac{(\Delta z)^{2}}{12} \frac{\partial^{2}}{\partial z^{2}}\left[\varepsilon_{0} \omega^{2} E_{x}(z, \omega)+\frac{1}{\mu_{0}} \frac{\partial^{2}}{\partial z^{2}} E_{x}(z, \omega)\right]\right|_{n, n_{\omega}} .
\end{aligned}
$$

Observe that the equation in the square brackets is the reduced wave equation or Helmholtz equation. When $E_{x}$ is an eigenfunction and $\omega$ is the corresponding eigenfrequency, the error will be zero as the homogeneous wave equation (inside square brackets) is zero. The criterion for obtaining optimal accuracy is that the net error of the discretized equation must be approximately zero whenever the operand is an eigenfunction and the frequency is equal to the corresponding eigenfrequency. Thus, when $E_{x}(z, t)=E_{x m}(z) \mathrm{e}^{-\mathrm{j} \omega_{m} t}, E_{x m}(z)$ is the $m^{\text {th }}$ eigenfunction of a mode with circular eigenfrequency $\omega_{m}$, the bracketed term, the homogeneous wave equation, and hence its derivatives will be zero. Transforming above equation back into the time domain results in

$$
\begin{aligned}
& \left(\delta A_{n, n_{t}}-\delta K_{n, n_{t}}\right) c_{n, n_{t}}= \\
& \left.\frac{(\Delta t)^{2}}{12} \frac{\partial^{2}}{\partial t^{2}}\left[\varepsilon_{0} \frac{\partial^{2}}{\partial t^{2}} E_{x}(z, t)-\frac{1}{\mu_{0}} \frac{\partial^{2}}{\partial z^{2}} E_{x}(z, t)\right]\right|_{n, n_{t}} \\
& +\left.\frac{(\Delta z)^{2}}{12} \frac{\partial^{2}}{\partial z^{2}}\left[\varepsilon_{0} \frac{\partial^{2}}{\partial t^{2}} E_{x}(z, t)-\frac{1}{\mu_{0}} \frac{\partial^{2}}{\partial z^{2}} E_{x}(z, t)\right]\right|_{n, n_{t}} .
\end{aligned}
$$

The above equation shows that the basic error of the modified operators is given by the derivatives of the homogeneous wave equation. Omitting the details of the derivation, the modified operators that yield errors of the above form are as follows

$$
\begin{aligned}
& A_{n, n_{t}}=\varepsilon_{0} \frac{1}{(\Delta t)^{2}} \begin{array}{|c|ccc|}
t+\Delta t & 1 / 12 & 10 / 12 & 1 / 12 \\
t & -2 / 12 & -20 / 12 & -2 / 12 \\
t-\Delta t & 1 / 12 & 10 / 12 & 1 / 12 \\
\hline & z-\Delta z & z & z+\Delta z \\
\hline
\end{array} \\
& K_{n, n_{t}}=\frac{1}{\mu_{0}} \frac{1}{(\Delta z)^{2}} \begin{array}{c|c|ccc|}
t+\Delta t & 1 / 12 & -2 / 12 & 1 / 12 \\
t & 10 / 12 & -20 / 12 & 10 / 12 \\
t-\Delta t & 1 / 12 & -2 / 12 & 1 / 12 \\
\hline & z-\Delta z & z & z+\Delta z \\
\hline
\end{array} \text {. }
\end{aligned}
$$

The homogeneous acoustic "A", elastic shear "E(S)", and elastic pressure "E(P)" 1-D wave equations read in the source-free case

$$
\begin{aligned}
& \text { A }\left(\rho_{\mathrm{a} 0} \frac{\partial^{2}}{\partial t^{2}}-\frac{1}{\kappa} \frac{\partial^{2}}{\partial z^{2}}\right) p(z, t)=0 \\
& \mathrm{E}(\mathrm{S}) \quad\left(\rho_{\mathrm{e} 0} \frac{\partial^{2}}{\partial t^{2}}-\mu \frac{\partial^{2}}{\partial z^{2}}\right) v_{x}(z, t)=0 \\
& \mathrm{E}(\mathrm{P}) \quad\left(\rho_{\mathrm{e} 0} \frac{\partial^{2}}{\partial t^{2}}-(\lambda+2 \mu) \frac{\partial^{2}}{\partial z^{2}}\right) v_{z}(z, t)=0,
\end{aligned}
$$

where in the "A" case $p$ is the pressure, $\rho_{\mathrm{a} 0}$ is the mass density at rest and $\kappa$ is the adiabatic compressibility; in the "E(S)" and " $\mathrm{E}(\mathrm{P})$ " case, representing the shear and pressure waves, $v_{x}, v_{z}$ are the particle velocities, $\rho_{\mathrm{e} 0}$ is the mass density at rest and $\lambda, \mu$ are Lamé's constants. Due to the similarity between the wave equations, all results mentioned above for $\mathrm{EM}$ case are also valid for $\mathrm{A}, \mathrm{E}(\mathrm{S})$ and $\mathrm{E}(\mathrm{P})$ cases.

\section{Modified operators for the 2-D TM case}

Consider the 2-D TM case in the $x z$ plane, then the sourcefree electromagnetic wave equation read

$$
\left(\varepsilon_{0} \frac{\partial^{2}}{\partial t^{2}}-\frac{1}{\mu_{0}}\left(\frac{\partial^{2}}{\partial x^{2}}+\frac{\partial^{2}}{\partial z^{2}}\right)\right) E_{y}(x, z, t)=0 .
$$

Discretizing the unknown field strength

$c_{p^{\prime} r^{\prime} N}=E_{y}\left(p^{\prime} \Delta x, r^{\prime} \Delta z, N \Delta t\right)$,

where $\Delta x, \Delta z$ represent the spatial grid spacing with respect to $x$ and $z$, and $\Delta t$ represents the temporal grid interval. The above equation can be written as

$$
\left(A_{p^{\prime} r^{\prime} N}^{1}-K_{p^{\prime} r^{\prime} N}^{1}-K_{p^{\prime} r^{\prime} N}^{3}\right) c_{p^{\prime} r^{\prime} N}=0 .
$$

$A_{p^{\prime} r^{\prime} N}^{1}, K_{p^{\prime} r^{\prime} N}^{1}, K_{p^{\prime} r^{\prime} N}^{3}$ are the temporal and spatial operators with respect to $x$ and $z$, respectively. The index $N$ represents the temporal grid points. The indices $p^{\prime}, r^{\prime}$ with $0 \leq p^{\prime} \leq N_{x}$, $0 \leq r^{\prime} \leq N_{z}$ represent the $x$ and $z$ directions, and $N_{x}$ and $N_{z}$ are the number of grid cells in $x$ and $z$ directions, respectively.

Using second-order central difference operators, the temporal operator error can be written as

$$
\left(\delta A_{p^{\prime} r^{\prime} N}^{1}\right)=\frac{(\Delta t)^{2}}{12} \frac{\partial^{2}}{\partial t^{2}}\left(\varepsilon_{0} \frac{\partial^{2}}{\partial t^{2}} E_{y}(x, z, t)\right) .
$$


In order to have optimal accuracy the operator error should be

$$
\begin{gathered}
\delta A_{p^{\prime} r^{\prime} N}^{1^{\prime}}=\left(\frac{(\Delta t)^{2}}{12} \frac{\partial^{2}}{\partial t^{2}}+\frac{(\Delta x)^{2}}{12} \frac{\partial^{2}}{\partial x^{2}}+\frac{(\Delta z)^{2}}{12} \frac{\partial^{2}}{\partial z^{2}}\right) \\
\times \varepsilon_{0} \frac{\partial^{2}}{\partial t^{2}} E_{y}(x, z, t) .
\end{gathered}
$$

Making use of the relation stated in Eq. (4) yields

$$
\begin{aligned}
A_{p^{\prime} r^{\prime} N}^{1^{\prime}}=(1+ & \left.\frac{(\Delta t)^{2}}{12} \frac{\partial^{2}}{\partial t^{2}}+\frac{(\Delta x)^{2}}{12} \frac{\partial^{2}}{\partial x^{2}}+\frac{(\Delta z)^{2}}{12} \frac{\partial^{2}}{\partial z^{2}}\right) \\
& \times \varepsilon_{0} \frac{\partial^{2}}{\partial t^{2}} E_{y}(x, z, t) .
\end{aligned}
$$

Neglecting higher order terms according to the second-order approximation the above equation can be approximated as

$A_{p^{\prime} r^{\prime} N}^{1^{\prime}}=\left(d_{p^{\prime} r^{\prime} N}\right)\left(f_{p^{\prime} r^{\prime} N}\right)\left(g_{p^{\prime} r^{\prime} N}\right)$

where $d$ is the operator for $\left(1+\frac{(\Delta t)^{2}}{12} \frac{\partial^{2}}{\partial t^{2}}\right) \varepsilon_{0} \frac{\partial^{2}}{\partial t^{2}} E_{y}, f$ is the operator for $\left(1+\frac{(\Delta x)^{2}}{12} \frac{\partial^{2}}{\partial x^{2}}\right)$ and $g$ is the operator for $\left(1+\frac{(\Delta z)^{2}}{12} \frac{\partial^{2}}{\partial z^{2}}\right)$. By neglecting higher order terms, any function $u(z)$ can be approximated as

$$
\begin{aligned}
\frac{u(z+\Delta z)-2 u(z)+u(z-\Delta z)}{(\Delta z)^{2}} & =\left(1+\frac{(\Delta z)^{2}}{12} \frac{\partial^{2}}{\partial z^{2}}\right) \frac{\partial^{2}}{\partial z^{2}} u \\
\frac{u(z+\Delta z)+10 u(z)+u(z-\Delta z)}{12} & =\left(1+\frac{(\Delta z)^{2}}{12} \frac{\partial^{2}}{\partial z^{2}}\right) u .
\end{aligned}
$$

Using the above equations, a modified temporal operator can be obtained. Following a similar approach, the spatial operators can also be obtained. Due to limited space, the modified operators are summarized at the end of this paper explicitly for an inhomogeneous medium.

In 2-D case and $x z$ plane, the homogeneous acoustic " $\mathrm{A}$ " and elastic shear "E(S)" wave equation read in the sourcefree case

$$
\begin{aligned}
& \left(\kappa \frac{\partial^{2}}{\partial t^{2}}-\frac{1}{\rho_{\mathrm{a} 0}}\left(\frac{\partial^{2}}{\partial x^{2}}+\frac{\partial^{2}}{\partial z^{2}}\right)\right) p_{y}(x, z, t)=0 \\
& \left(\rho_{\mathrm{e} 0} \frac{\partial^{2}}{\partial t^{2}}-\mu\left(\frac{\partial^{2}}{\partial x^{2}}+\frac{\partial^{2}}{\partial z^{2}}\right)\right) v_{y}(x, z, t)=0 .
\end{aligned}
$$

Due to the similarity between the wave equations, all results mentioned above for the electromagnetic waves are also valid for the acoustic and elastic waves.

\section{Applicability to staggered grid approaches (FDTD, FIT)}

In a homogeneous medium the first-order staggered grid approach consists of solving the following coupled first-order equations in differential form

$$
\begin{aligned}
\frac{\partial}{\partial t} H_{y}(z, t) & =-\frac{1}{\mu_{0}} \frac{\partial}{\partial z} E_{x}(z, t) \\
\frac{\partial}{\partial t} E_{x}(z, t) & =-\frac{1}{\varepsilon_{0}} \frac{\partial}{\partial z} H_{y}(z, t)-\frac{1}{\varepsilon_{0}} J_{e x}(z, t) .
\end{aligned}
$$

For the sake of simplicity, subscripts are dropped from here onwards. Using a Taylor's series expansion in Eq. (24) yields

$$
\begin{aligned}
& \frac{\partial}{\partial t} H(z, t)+\frac{(\Delta t)^{2}}{24} \frac{\partial^{2}}{\partial t^{2}} \frac{\partial}{\partial t} H(z, t) \\
& \quad=-\frac{1}{\mu_{0}}\left(\frac{\partial}{\partial z} E(z, t)+\frac{(\Delta z)^{2}}{24} \frac{\partial^{2}}{\partial z^{2}} \frac{\partial}{\partial z} E(z, t)\right) .
\end{aligned}
$$

To satisfy the general criterion for optimally accurate operators the Taylor's series should be

$$
\begin{aligned}
& \frac{\partial}{\partial t} H(z, t)+\frac{(\Delta t)^{2}}{24} \frac{\partial^{2}}{\partial t^{2}}\left[\frac{\partial}{\partial t} H(z, t)+\frac{1}{\mu_{0}} \frac{\partial}{\partial z} E(z, t)\right]= \\
& -\frac{\frac{\partial}{\partial z} E(z, t)+\frac{(\Delta z)^{2}}{24} \frac{\partial^{2}}{\partial z^{2}}\left[\frac{\partial}{\partial z} E(z, t)-\mu_{0} \frac{\partial}{\partial t} H(z, t)\right]}{\mu_{0}} .
\end{aligned}
$$

The derivation of modified operators from the above equation is possible, but they apparently lead to an intractable implicit scheme. Thus, the modified operators formally exist but their use would lead to an apparently intractable implicit scheme.

\section{Implementation}

Let the following equation represent a discretized wave equation using modified operators

$$
\left(A_{n, n_{t}}-K_{n, n_{t}}\right) c_{n, n_{t}}=f,
$$

where here for the sake of completeness, even the source term is also considered. From Eqs. (9) and (10), the modified operator $\left(A_{n, n_{t}}-K_{n, n_{t}}\right)$ has multiple non-zero elements for time $t+\Delta t$. Usage of these operators in the marchingon-in-time scheme to solve the FD equation results in an implicit scheme, rather than an explicit scheme. To obviate the need to solve a system of simultaneous linear equations at each time step, the modified operators are approximated by a predictor-corrector scheme based on the first-order Born approximation. The FD equation using the conventional operators can be written as

$$
\left(A_{n, n_{t}}^{0}-K_{n, n_{t}}^{0}\right) c_{n, n_{t}}^{0}=f
$$

where $f$ is the source term. First, predict the field at the next time step using the conventional operators $A^{0}$ and $K^{0}$. Compute $\delta c$, the correction to the displacement at time $t+$ $\Delta t$, using the first-order Born approximation. To obtain the correction solve

$$
\left(A_{n, n_{t}}^{0}-K_{n, n_{t}}^{0}\right) \delta c_{n, n_{t}}=-\left(\delta A_{n, n_{t}}-\delta K_{n, n_{t}}\right) c_{n, n_{t}}^{0} .
$$



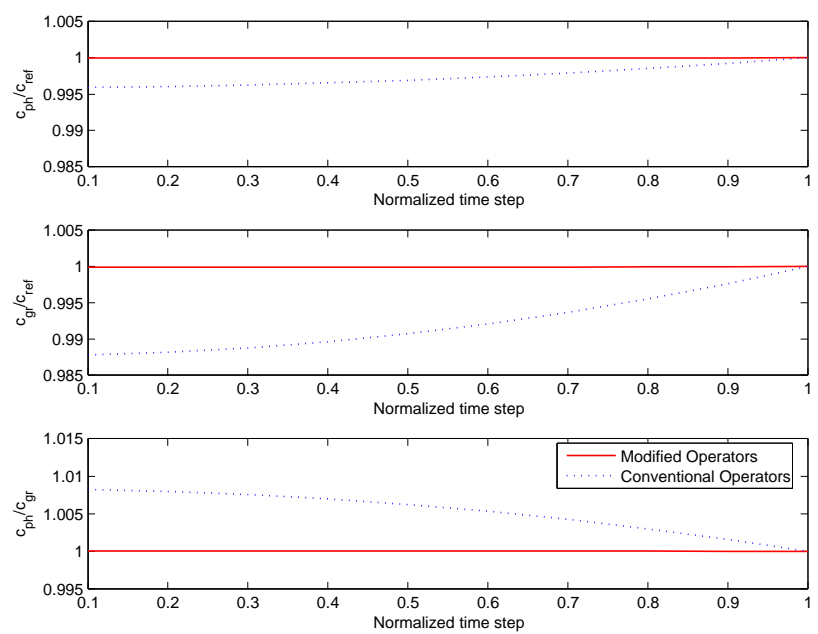

Fig. 1. Dispersion relation graphs.

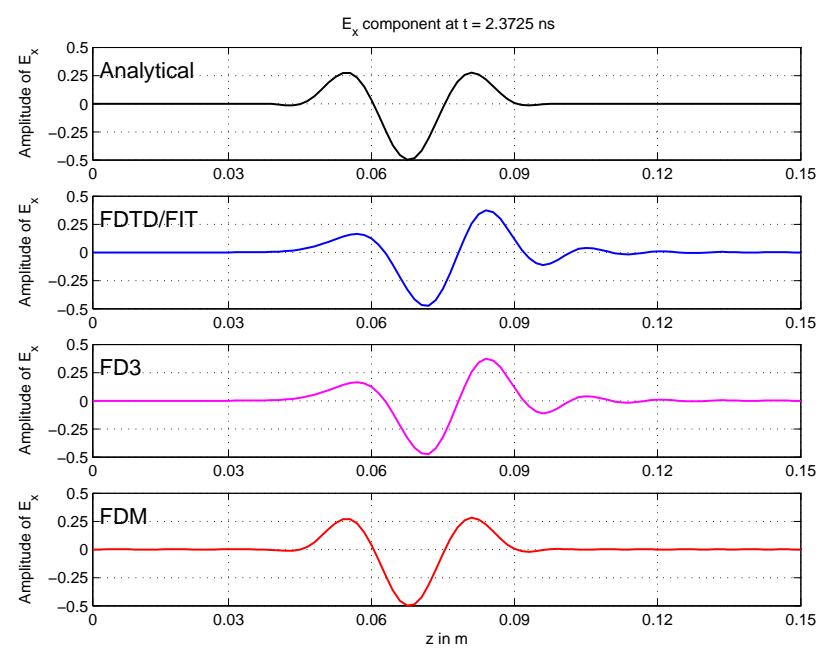

Fig. 2. Comparison between the analytical and numerical solutions.

The operators $\delta A_{n, n_{t}}, \delta K_{n, n_{t}}$ denote the difference between the modified operators $A_{n, n_{t}}, K_{n, n_{t}}$ and the conventional operators $\left.A_{n, n_{t}}^{0}, K_{n, n_{t}}^{0}\right)$. The left-hand side of the above equation uses conventional operators, this means $\delta c_{n, n_{t}}$ can be obtained explicitly at time $t+\Delta t$. Compute the corrected field after each time step using $c_{n, n_{t}}^{0}$ and $\delta c_{n, n_{t}}$ computed above, i.e., $c_{n, n_{t}+1}=c_{n, n_{t}+1}^{0}+\delta c_{n, n_{t}+1}$. Before advancing to the next time step redefine $c_{n, n_{t}+1}^{0} \leftarrow c_{n, n_{t}+1}$.

\section{Simulation results}

\subsection{Dispersion relation graphs}

Figure 1 shows the variation of numerical phase velocity and group velocity with respect to the normalized time step, $\widehat{\Delta t}$, for a given spatial resolution, number of cells per wavelength, in this case 20. Modified operators have less depen-

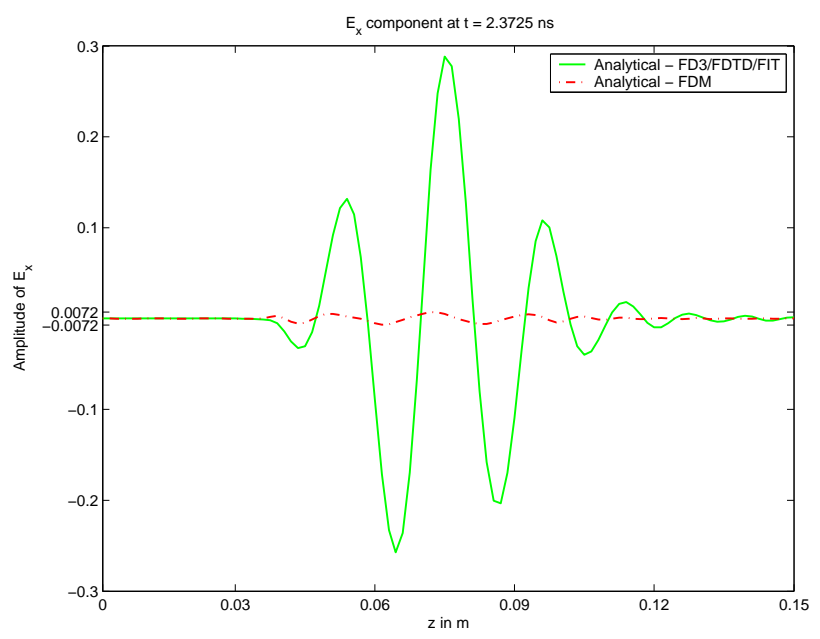

Fig. 3. Absolute difference between the analytical and numerical solutions.

dency on the normalized time step, and also have a sharper characteristics than standard operators. This is clearly visible as numerical phase and group velocities of modified operators tend to be always the free-space wave velocity, i.e., $c_{\mathrm{ph}}=c_{\mathrm{gr}}=c_{0}$.

\subsection{1-D EM case: Free space modeling: PEC boundaries}

All the simulations, see Figs. 2 and 3, are carried out in freespace of the length $1.5 \mathrm{~m}, 1000$ cells, for $2.3725 \mathrm{~ns}, 950$ time steps, with a raised cosine pulse of 2 cycles, $\mathrm{RC}_{2}$ pulse, with a carrier frequency of $10 \mathrm{GHz}$ as the source located at the center of the medium. The normalized time step $\widehat{\Delta t}$ is equal to 0.5 , the reference cell size $\Delta z$ and reference time step $\Delta t$ are $1.5 \mathrm{~mm}$ and $5 \mathrm{ps}$, respectively. The spatial resolution is taken as 20. The results are plotted from $z=0, \ldots, 0.15 \mathrm{~m}$. Figurre 2 shows a comparative plot of the analytical and numerical solutions. The numerical dispersion error is clearly visible in Fig. 3, displays the difference between the analytical and numerical solutions. The numerical dispersion error is reduced approximately by a factor of 40 .

\subsection{2-D TM case: inhomogeneous medium/plane wave boundaries}

The 2-D modeling results in Figs. 4 and 5 are obtained by simulations carried out in an area of a length of $3 \mathrm{~m}$, 2000 cells, and a width of $1.5 \mathrm{~m}, 1000$ cells, for $10 \mathrm{~ns}$, 4000 time steps, with a raised cosine pulse of 2 cycles, $R_{2}$ pulse, with a carrier frequency of $10 \mathrm{GHz}$ as source located along the top boundary of the medium. The normalized time step $\widehat{\Delta t}$ is equal to 0.5 , the reference cell size $\Delta z$ and reference time step $\Delta t$ are $1.5 \mathrm{~mm}$ and $5 \mathrm{ps}$, respectively. The spatial resolution is taken as 20. A plane wave boundary condition is used to truncate the simulation. The first medium $(0-2.25 \mathrm{~m})$ is free space and the second medium $(2.25-3 \mathrm{~m})$ has an increase in permittivity by a factor of 4 . A plane wave 


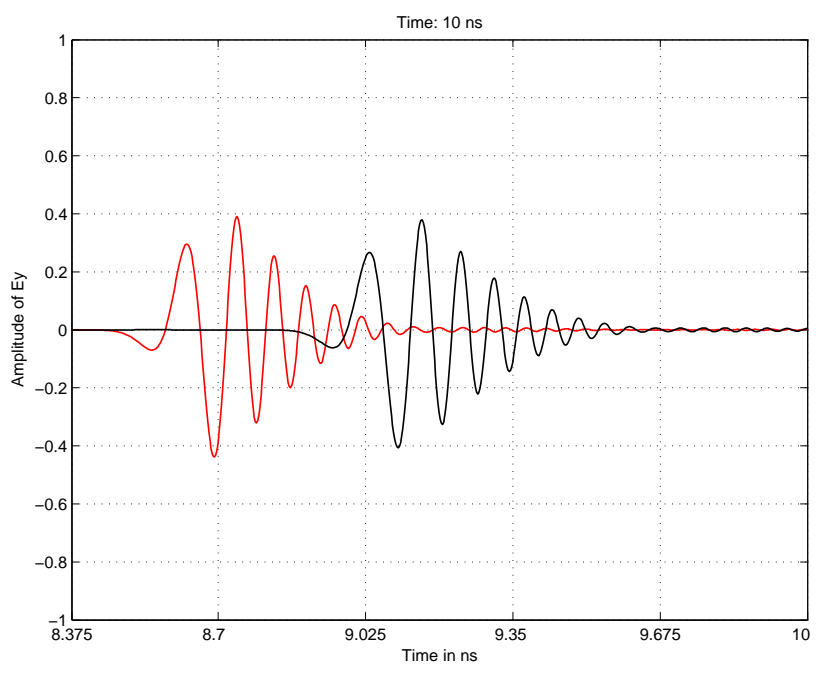

Fig. 4. Wave modeling: FIT.

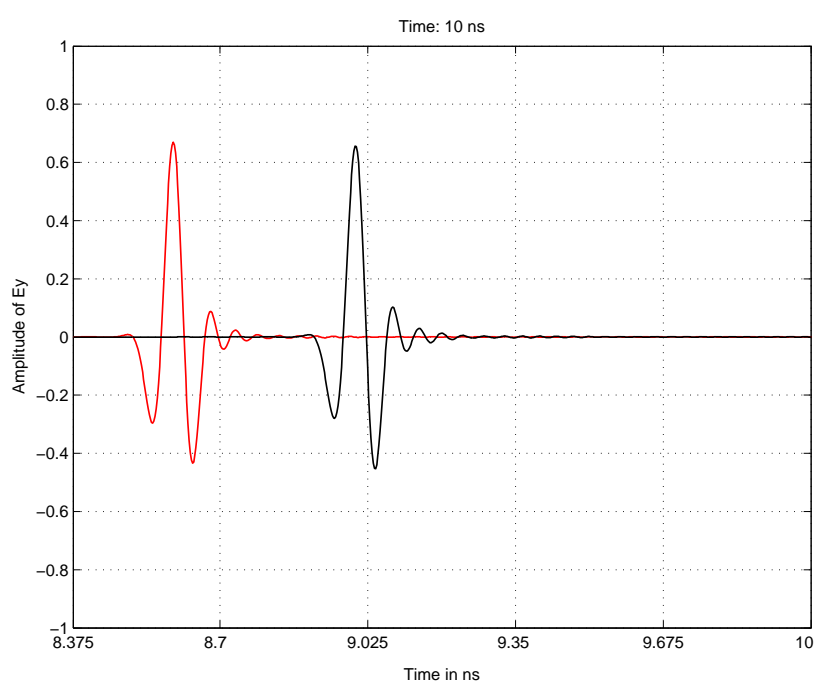

Fig. 5. Wave modeling: FDM.

is sent from the top of the medium and is observed as it passes through the second medium. Figures 4 and 5 shows the data collected by the receivers (inside the second medium). From these figures it clear that FDM outweighs FIT even in the case of an inhomogeneous problem.

\subsection{2-D P-SV case: plexiglas/motion-free boundaries}

Consider the results in Fig. 6 where the simulations are carried out for plexiglas with an area of length of $66.2266 \mathrm{~mm}$, 1200 cells, and a width of $132.4535 \mathrm{~mm}, 2000$ cells, for a time interval of $90.9707 \mu \mathrm{s}, 6500$ time steps, with a raised cosine pulse of 2 cycles, $\mathrm{RC}_{2}$ pulse, with a carrier frequency of $5 \mathrm{MHz}$ as source. The normalized time step $\widehat{\Delta t}$ is equal
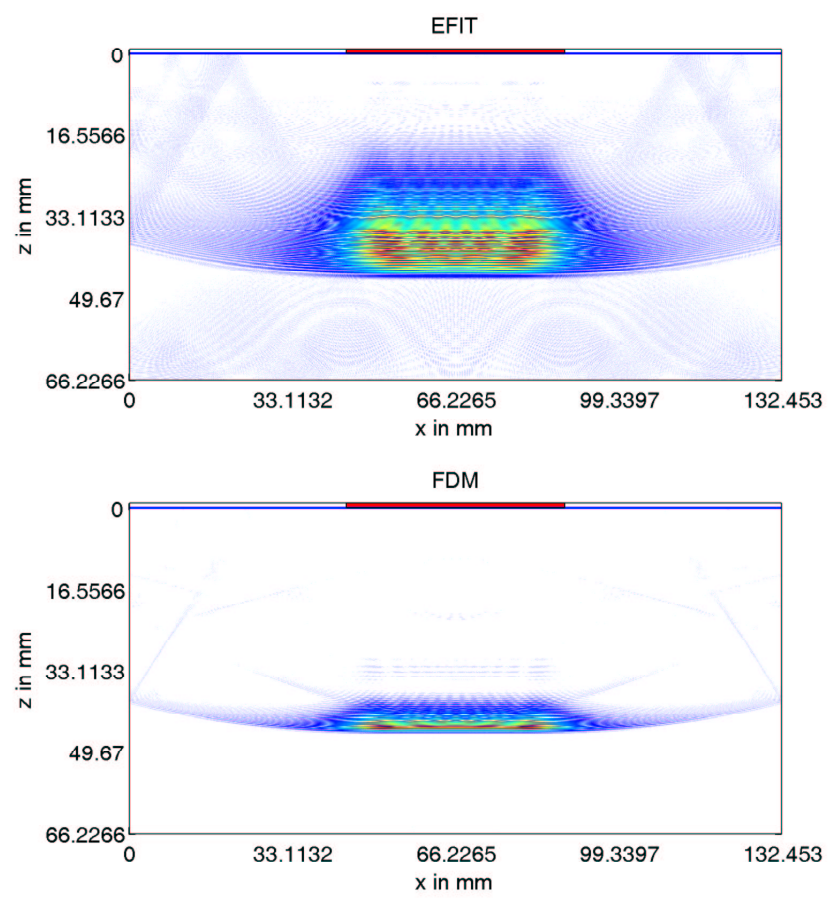

Fig. 6. Elastic P-SV wave modeling using EFIT and FDM.

to 0.5 . The reference cell size $\Delta z$ and reference time step $\Delta t$ are $55.189 \mu \mathrm{m}$ and $20.2157 \mathrm{~ns}$, respectively. The spatial resolution, number of grid cells per wavelength, is taken as 9.8933. The motion-free boundary condition is used to truncate the simulation on all sides. A normal force is applied within a finite aperture at the top of the medium. The finite aperture is shown in red color. The EFIT and FDM snapshot of the wave field is taken at $t=90.9707 \mu \mathrm{s}$. In this case, using FDM, accuracy is increased by a factor of 30 compared to EFIT.

\section{Conclusions}

The errors due to spatial and temporal discretization cannot be considered separately. The net error due to the combined effects of temporal and spatial discretization must be considered as a single quality. This error can be minimized by tuning the operators so that the errors due to spatial and temporal discretization come as close as possible to cancel each other. The error of the conventional operators is much worse than that of the modified operators, but approaches that of modified operators if and only if $\widehat{\Delta t}$ is equal to the Courant limit. The error of solutions obtained using modified operators is essentially uniform regardless of the choice of $\widehat{\Delta t}$ and spatial resolution. This is clear from the dispersion graphs. We make the following observations: 
- a error reduction by a factor of about $40-100$ for 2 times CPU requirement compared to FD3 method in $1-\mathrm{D}$ case

- a error reduction by a factor of about 36 for 2.9 times CPU requirement compared to FD3 method in 2-D TM (SH) case, and

- a error reduction by a factor of about 30 , for 3.5 times additional CPU requirement compared to FD3 method in 2-D P-SV case.

Please note that the reduction of error depends on the complexity of the problem, too. This method is straightly extendable to inhomogeneous problems and also to the 3-D problems. The ratio of required floating point operations will increase linearly with the dimension of the problem and a CPU time of about 5-8 times can be expected. An improvement of the results by a factor of 30 can be expected as the improvement in the accuracy is independent of the dimension of the problem. The P-SV case is not furnished here, but it can be treated in a similar way, which is part of our present research.

\section{Appendix}

$K_{p^{\prime} r^{\prime} N}^{1^{\prime}}=\left(\frac{1}{144(\Delta x)^{2}}\right) *$

\begin{tabular}{|c|c|c|c|c|}
\hline \multirow[t]{2}{*}{$t+\Delta t$} & $\begin{array}{c}z+\Delta z \\
z \\
z-\Delta z\end{array}$ & $\begin{array}{c}v_{-0} \\
10 v_{-0} \\
v_{-0} \\
\end{array}$ & $\begin{array}{c}-v_{-+} \\
-10 v_{-+} \\
-v_{-+}\end{array}$ & $\begin{array}{c}v_{+0} \\
10 v_{+0} \\
v_{+0}\end{array}$ \\
\hline & & $x-\Delta x$ & $x$ & $x+\Delta x$ \\
\hline \multirow[t]{2}{*}{$t$} & $\begin{array}{c}z+\Delta z \\
z \\
z-\Delta z\end{array}$ & $\begin{array}{c}v_{-0} \\
10 v_{-0} \\
v_{-0}\end{array}$ & $\begin{array}{l}-10 v_{-+} \\
-100 v_{-+} \\
-10 v_{-+}\end{array}$ & $\begin{array}{c}v_{+0} \\
10 v_{+0} \\
v_{+0}\end{array}$ \\
\hline & & $x-\Delta x$ & $x$ & $x+\Delta x$ \\
\hline \multirow[t]{2}{*}{$t-\Delta t$} & $\begin{array}{c}z+\Delta z \\
z \\
z-\Delta z\end{array}$ & $\begin{array}{c}v_{-0} \\
10 v_{-0} \\
v_{-0}\end{array}$ & $\begin{array}{c}-v_{-+} \\
-10 v_{-+} \\
-v_{-+}\end{array}$ & $\begin{array}{c}v_{+0} \\
10 v_{+0} \\
v_{+0}\end{array}$ \\
\hline & & $x-\Delta x$ & $x$ & $x+\Delta x$ \\
\hline
\end{tabular}

The indices $p^{\prime}, r^{\prime}$ represent the $x$ and $z$ directions respectively, $0 \leq p^{\prime} \leq N_{x}, 0 \leq r^{\prime} \leq N_{z} . N_{x}$ and $N_{z}$ are the number of grid cells in $x$ and $z$ directions. In spatial operators, $\frac{1}{\mu}$ is represented as $v$. $v_{-0}=\frac{\left(v_{\left(p^{\prime}-1\right) r^{\prime}}+v_{p^{\prime} r^{\prime}}\right)}{2}$

$v_{+0}=\frac{\left(v_{\left(p^{\prime}+1\right) r^{\prime}}+v_{p^{\prime} r^{\prime}}\right)}{2}$

$v_{-+}$represents the sum of $v_{-0}$ and $v_{+0}$.

$K_{p^{\prime} r^{\prime} N}^{3^{\prime}}=\left(\frac{1}{144(\Delta z)^{2}}\right) *$

\begin{tabular}{|c|c|c|c|c|}
\hline \multirow[t]{2}{*}{$t-\Delta t$} & $\begin{array}{c}z+\Delta z \\
z \\
z-\Delta z \\
\end{array}$ & $\begin{array}{c}v_{0-} \\
-v_{-+} \\
v_{0+} \\
\end{array}$ & $\begin{array}{c}v_{0-} \\
-10 v_{-+} \\
v_{0+} \\
\end{array}$ & $\begin{array}{c}v_{0-} \\
-v_{0-+} \\
v_{0+} \\
\end{array}$ \\
\hline & & $x-\Delta x$ & $x$ & $x+\Delta x$ \\
\hline \multirow[t]{2}{*}{$t$} & $\begin{array}{c}z+\Delta z \\
z \\
z-\Delta z\end{array}$ & $\begin{array}{c}10 v_{0-} \\
-10 v_{-+} \\
10 v_{0+}\end{array}$ & $\begin{array}{c}10 v_{0-} \\
-100 v_{-+} \\
10 v_{0+}\end{array}$ & $\begin{array}{c}10 v_{0-} \\
-10 v_{-+} \\
10 v_{0+}\end{array}$ \\
\hline & & $x-\Delta x$ & $x$ & $x+\Delta x$ \\
\hline \multirow[t]{2}{*}{$t-\Delta t$} & $\begin{array}{c}z+\Delta z \\
z \\
z-\Delta z\end{array}$ & $\begin{array}{c}v_{0-} \\
-v_{-+} \\
v_{0+}\end{array}$ & $\begin{array}{c}v_{0-} \\
-10 v_{-+} \\
v_{0+}\end{array}$ & $\begin{array}{c}v_{0-} \\
-v_{0-+} \\
v_{0+}\end{array}$ \\
\hline & & $x-\Delta x$ & $x$ & $x+\Delta x$ \\
\hline
\end{tabular}

$v_{0-}=\frac{\left(v_{p^{\prime}\left(r^{\prime}-1\right)}+v_{p^{\prime} r^{\prime}}\right)}{2}$

$v_{0+}=\frac{\left(v_{p^{\prime}\left(r^{\prime}+1\right)}+v_{p^{\prime} r^{\prime}}\right)}{2}$

Here $v_{-+}$represents the sum of $v_{0-}$ and $v_{0+}$.

$A_{p^{\prime} r^{\prime} N}^{1^{\prime}}=\left(\frac{\varepsilon_{00}}{144(\Delta t)^{2}}\right) *$

\begin{tabular}{|c|c|c|c|c|}
\hline \multirow[t]{2}{*}{$t+\Delta t$} & $\begin{array}{c}z+\Delta z \\
z \\
z-\Delta z\end{array}$ & $\begin{array}{c}1 \\
10 \\
1 \\
\end{array}$ & $\begin{array}{c}10 \\
100 \\
10 \\
\end{array}$ & $\begin{array}{c}1 \\
10 \\
1 \\
\end{array}$ \\
\hline & & $x-\Delta x$ & $x$ & $x+\Delta x$ \\
\hline \multirow[t]{2}{*}{$t$} & $\begin{array}{c}z+\Delta z \\
z \\
z-\Delta z\end{array}$ & $\begin{array}{c}-2 \\
-20 \\
-2 \\
\end{array}$ & $\begin{array}{c}-20 \\
-200 \\
-20 \\
\end{array}$ & $\begin{array}{c}-2 \\
-20 \\
-2 \\
\end{array}$ \\
\hline & & $x-\Delta x$ & $x$ & $x+\Delta x$ \\
\hline \multirow{4}{*}{$t-\Delta t$} & $z+\Delta z$ & 1 & 10 & 1 \\
\hline & $z$ & 10 & 100 & 10 \\
\hline & $z-\Delta z$ & 1 & 10 & 1 \\
\hline & & $x-\Delta x$ & $x$ & $x+\Delta x$ \\
\hline
\end{tabular}

where $\epsilon_{00}$ represents $\epsilon_{p^{\prime} r^{\prime}}$. 


\section{References}

Bommaraju, C.: Optimally accurate second-order time-domain finite-difference scheme for the acoustic, electromagnetic and elastodynamic wave modeling, M.Sc. Thesis, University of Kassel, Kassel, Germany, 2004.

Bommaraju, C. and Marklein, R.: Optimally accurate second-order time-domain finite-difference scheme for the acoustic, electromagnetic and elastodynamic wave modeling: 1-D case, Proc. of IEEE Intern. Symposium on Antennas and Propagation and USNC/URSI National Radio Science Meeting, 2004.

Geller, R. J. and Takeuchi, N.: A new method for computing highly accurate DSM synthetic Seismograms, Geophysics Journal International, 123, 449-470, 1995.
Geller, R. J. and Takeuchi, N.: Optimally accurate second-order time-domain finite difference scheme for the elastic equation of motion: one-dimensional case, Geophysics Journal International, 135, 48-62, 1998.

Geller, R. J. and Takeuchi, N.: Optimally accurate second-order time-domain finite difference scheme for computing synthetic seismograms in 2-D and 3-D media, Physics of the Earth and Planetary Interiors, 119, 99-131, 2000.

Marklein, R.: Numerical Methods for the Modeling of Acoustic, Electromagnetic, Elastic and Piezoelectric Wave Propagation Problems in the Time Domain Based on the Finite Integration Technique (in German), Shaker Verlag, Aachen, Germany, 1997.

Taflove, A. and Hagness, S. C.: Computational Electrodynamics: The Finite-Difference Time-Domain Method, 2nd ed., Artech House, Boston, 2000. 